\title{
Novas técnicas cirúrgicas para o tratamento da atresia pulmonar com comunicação interventricular e anomalias de artérias pulmonares incluindo o assim chamado truncus tipo IV
}

Miguel BARBERO-MARCIAL*, Edmar ATIK*, Miguel RATTI*, Luiz Junya KAJITA*, Arlindo RISO*, Geraldo VERGINELLI*, Delmont BITTENCOURT*, Fulvio PILEGGI*, Adib D. JATENE*

\author{
RBCCV
}

BARBERO-MARCIAL, M.; ATIK, E.; RATTI, M.; KAJITA, L.J.; RISO, A.; VERGINELLI, G.; BITTENCOURT, D.; PILEGGI, F.; JATENE, A.D. - Novas técnicas cirúrgicas para o tratamento da atresia pulmonar com comunicação interventricular e anomalias de artérias pulmonares incluindo o assim chamado truncus tipo IV. Rev. Bras. Circ. Cardiovasc., 2(1):22-31, 1987

RESUMO: Entre janeiro de 1975 e outubro de 1986, 42 pacientes com atresia pulmonar e comunicação interventricular, com idade entre 2 e 18 anos, foram submetidos a correção parcial, ou total. Foram divididos em: tipo A) com todos os segmentos broncopulmonares conectados às artérias pulmonares (AP's), 34 pacientes; tipo B) com alguns dos segmentos broricopulmonares conectados às AP's, 6 pacientes; tipo C) com todos os segmentos broncopulmonares conectados às colaterais sistêmico-pulmonares, 2 pacientes. A correção foi planejada em uma a três etapas. No tipo A, 17 foram corrigidos em uma etapa, com três óbitos; em 9, na primeira etapa, as AP's foram reconstruidas e o Blalock (BT), realizado, tendo ocorrido um óbito. Em 2, a segunda etapa de correção total foi realizada, sem óbitos. No tipo B, a primeira etapa de unificação das colaterais intra ou extra-hilares foi realizada em 6 casos, sem óbitos; em 2, a segunda etapa da correçāo total foi realizada, com um óbito. No tipo C, 2 pacientes foram operados; 1 em três etapas; a primeira constou de construção de segmento arterial intermediário entre as artérias lobares e - BT; a segunda compreendeu unificação das colaterais contralaterais e a terceira, restabelecimento da continuidade ventrículo direito - circulação pulmonar; o paciente teve boa evolução. No segundo caso, a correção foi realizada após somente uma intervenção prévia. A evolução foi satisfatória. Estudos hemodinâ. micos seriados foram realizados em 32 pacientes. As técnicas propostas permitem obter condiçōes para correção total com adequada relação pós-operatória das pressões ventrículo direito - ventrículo esquerdo.

DESCRITORES: atresia pulmonar, cirurgia; comunicação interventricular, cirurgia; artérias pulmonares, anomalias, cirurgia.

\section{INTRODUÇĀO}

O tratamento cirúrgico da atresia pulmonar com comunicação interventricular (AP C/CIV) tem sido bem sucedido, na maioria dos pacientes que possuem boas artérias pulmonares intrapericárdicas (AP's) e boa distribuição periférica ${ }^{1,711}$. Nestes casos, a correção cirúrgica do defeito do septo interventricular, seguida do restabelecimento da continuidade entre o ventrículo direito (VD) e AP's com

Trabalho realizado no Instituto do Coração do Hospital das Clínicas da Faculdade de Medicina da Universidade de São Paulo. São Paulo, SP. Brasil.

Apresentado ao 14: Congresso Nacional de Cirurgia Cardiaca, Salvador, BA, 27 e 28 de março, 1987.

- Do Instituto do Coração do Hospital das Clínicas da Faculdade de Medicina da Universidade de São Paulo.

Endereço para separatas: Miguel Barbero-Marcial. Av. Dr. Enéias de Carvalho Aguiar, 44 - 2 andar, Divisão Cirúrgica, 05403 - São Paulo, SP. Brasil. 
um tubo valvulado, tem permitido um bom resultado hemodinâmico, com pressões sistólicas no VD próximas ao normal. No entanto, quando as AP's são hipoplásicas, ou têm segmentos estenóticos, ou existe descontinuidade, a correçāo cirúrgica tornase impossivel, ou, quando realizada, os resultados são pouco satisfatórios. O mesmo acontece quando a AP C/CIV se acompanha de anormalidades na arborização pulmonar ${ }^{12}{ }^{14}{ }^{15}$, ou quando não existem evidências de AP's extraparenquimatosas e toda a circulação pulmonar é mantida por colaterais aorto-pulmonares ${ }^{1,7,9,11,13,17}$.

Em 1986, propusemos ${ }^{2}$ a seguinte classificação, que, considerando o exposto anteriormente, nos parece de valor clínico e cirúrgico.

AP C/CIV tipo A (clássico): todos os segmentos broncopulmonares (SBP) estão conectados com as AP's centrais, sejam estas normais, ou não. Tipo B (misto): alguns dos SBP estão conectados com as AP's centrais e outros, com artérias colaterais sistêmico-pulmonares. Tipo C: todos os SBP são conectados às colaterais sistêmico-pulmonares, sem evidências de tronco pulmonar ou AP's centrais (também chamado truncus tipo IV).

Este trabalho enfatizará as novas técnicas cirúrgicas propostas para o tratamento de atresias pulmonares com CIV complexas, quais sejam as associadas a estenose, ou descontinuidade das AP's e as que possuem anormalidades parciais, ou totais da distribuição periférica arterial pulmonar.

\section{CASUÍSTICA E MÉTODOS}

Entre janeiro de 1975 e fevereiro de 1987, 45 pacientes com diagnóstico de base de AP C/CIV, com idade entre dois meses e dezoito anos, foram submetidos a correção parcial ou total, em uma, ou até três etapas cirúrgicas. Para facilitar a exposição, os pacientes serão divididos em três grupos, seguindo a classificação acima proposta:

Tipo A: Todos os SBP conectados às AP's centrais; este grupo foi subdividido em dois subgrupos:

$A_{1}$ ) Sem anormalidades das AP's intra ou extrapericárdicas.

Dezessete pacientes, com idade variável entre dois e doze anos, foram submetidos a correção cirúrgica.

Destes casos, 8 tinham operação de BlalockTaussig prévia (sendo que, em 2 pacientes, duas anastomoses foram realizadas); 7 tinham ducto arterioso e 1, múltiplas colaterais. A correção cirúr- gica seguiu a já previamente estabelecida na literatura $^{7}$. O tubo utilizado, em 14 casos, foi o de woven Dacron valvulado (prótese de dura-máter, ou de pericárdio bovino); em 3 pacientes, a atresia era somente infundibular ou ânulo-valvar e a correção foi realizada mediante ressecção e ampliação da região atrésica.

Dado que, neste grupo não foram utilizadas novas técnicas cirúrgicas, o que constitui o motivo deste trabalho e que não serão comentadas mais adiante, os resultados serão expostos a seguir. Um paciente faleceu, no $8^{\circ}$ dia de pós-operatório, por septicemia; 1 paciente faleceu 24 horas depois da operação, após parada cardíaca súbita, interpretada como provável compressão do tubo de Dacron sobre a artéria descendente anterior, que, nascendo da coronária direita, atravessa o infundíbulo; o terceiro paciente apresentou quadro de baixo débito progressivo, interpretado, na ocasião, como secundário à falência miocárdica. Todos os pacientes tiveram boa evolução pós-operatória.

\section{$\mathrm{A}_{2}$ ) Com anomalias das AP's}

Nove pacientes* consecutivos, com idade variável entre três meses e dois anos, portadores de AP com CIV e estenose segmentar, ou descontiuidade das AP's, foram submetidos, simultaneamente, à operação de Blalock-Taussig (clássica, ou modificada) e correção da estenose, ou reconstrução para estabelecimento da continuidade das artérias pulmonares.

Todos os casos necessitaram de procedimento paliativo, para evitar crises de anóxia, ou para diminuir a cianose. As anomalias das AP's foram: artéria pulmonar direita (APD) nascendo do ductus, com ausência angiográfica da esquerda (1 caso); estenose segmentar da artéria pulmonar esquerda (APE) (2 casos); estenose justaductal da APE e da APD (3 casos); hipoplasia do tronco pulmonar (TP) e da APD, nascendo a APE do ductus arteriosus (2 casos); hipoplasia do TP e da APD, sendo a APE hipoplásica e em situação intraparenquimatosa (1 caso).

\section{Técnica operatória}

Todas as operaçōes foram realizadas sem circulação extracorpórea. Em razão da atresia pulmonar, quando o ductus teve que ser ocluído durante a reconstrução, a operação de Blalock-Taussig foi realizada primeiramente, para evitar hipóxia; após isto, a artéria pulmonar proximal foi fechada e o reparo, realizado.

\footnotetext{
- Seis destes pacientes fazem parte da série original de pacientes submetidos a reconstruçâo, em publicação no $\mathrm{J}$. Thorac. Cardiovasc. Surg
} 
I - Estenose segmentar das artérias pulmonares (Figura 1). As seguintes técnicas foram utilizadas:

la - Dilatação do segmento estenosado (1 caso); o paciente não apresentava evidência angiográfica de APE e a APD era continuação do ducto arterioso. Apesar disto, a toracotomia esquerda foi realizada e a APE foi identificada e aberta; não existia sangramento proximal e uma pequena quantidade de trombos organizados foram retirados. Após a dilatação com probes de tamanho crescente de 1 a $5 \mathrm{~mm}$ e a obtenção de um adequado fluxo proximal, o Blalock modificado foi realizado.

lb - Ampliação do segmento estenótico. Nos pacientes com hipoplasia segmentar da APE, a técnica empregada foi a seguinte: a) No primeiro paciente, através de toracotomia esquerda, o Blalock modificado foi realizado e, após a obtenção adequada de $\mathrm{paO}_{2}$, uma pinça curva foi colocada no lado esquerdo do TP, na altura da origem da APE. Uma segunda pinça foi colocada imediatamente proximal à anastomose do Blalock e uma terceira no ducto arterioso. Em seguida, uma incisão longitudinal foi realizada na APE, começando distalmente ao ducto, passando através do segmento estenosado, até o TP. Para ampliar o segmento, um retaIho de PTFE foi usado. b) No segundo paciente, através de esternotomia mediana, foi realizada a operação de Blalock-Taussig modificado, entre a artéria subclávia direita e a APD e, a seguir, ampliação da APE com pericárdio autógeno com o fechamento do ducto.
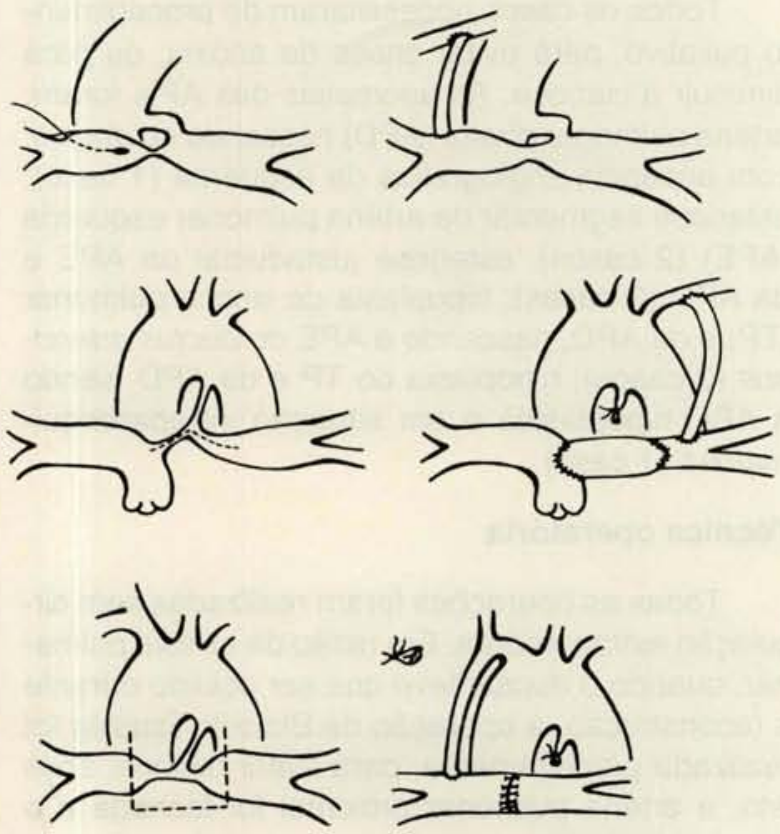

Fig. 1 - Representaçâo esquemática da técnica operatória. A: dilataçăo do segmento estenosado. B: ampliação do segmento estenosado. C: ressecçăo do segmento estenosado e anastomose término-terminal.
Ic - Ressecção do segmento estenosado e anastomose término-terminal. Em 1 paciente, através de toracotomia esquerda, foi realizada a operação de Blalock-Taussig modificado, no segmento distal da APE; a seguir, o segmento estenótico da APE foi isolado e ressecado, assim como o ducto arterioso; o remanescente do ducto foi suturado ao nível da aorta e uma anastomose términoterminal com pontos separados foi realizada entre o segmento proximal e o distal da APE. Em 2 pacientes, a abordagem foi por esternotomia mediana; o Blalock clássico para a APD foi feito e, a seguir, adequados os níveis de $\mathrm{paO}_{2}$ e condições hemodinâmicas satisfatórias, o segmento juxtaductal da APD e APE foi ressecado, o ducto, fechado e a anastomose término-terminal com pontos separados $7-0$ foi realizada.

II - Artérias pulmonares não confluentes (Figura 2).

Três pacientes apresentaram hipoplasia moderada do tronco pulmonar e da APD, com a APE originando-se do ducto arterioso. Foi realizada ressecção da porção da APE relacionada ao ductus e à anastomose entre esta artéria e o TP. A bandagem foi feita por esternotomia mediana; uma operação de Blalock modificado foi realizada entre a artéria subclávia direita e a APD com tubo PTFE de $5 \mathrm{~mm}$; a seguir, foi realizada a dissecção extra e intra-hilar da APE, para obter-se mobilização adequada. O segmento estenótico da APE juxtaductal foi ressecado e a anastomose da APE com a parede superior e lateral do TP foi feita.

Tipo $B$ (misto): Alguns dos segmentos broncopulmonares conectados às AP's centrais e outros com artérias colaterais sistêmico-pulmonares.

Nove pacientes, com idade variável entre quatro meses e sete anos, foram submetidos a correção em etapas. Nos 9, a primeira etapa, de unificação de uma ou mais colaterais com a artéria pulmonar do mesmo lado, foi realizada; em 3 deles, após período variável entre seis e vinte e três meses, foi feito o restabelecimento da continuidade entre o ventrículo direito e a circulação pulmonar, após o fechamento do defeito interventricular.

As anomalias de arborização pulmonar foram:

Em 4 pacientes, a APE irrigava somente segmentos broncopulmonares do lobo superior esquerdo e as colaterais irrigavam o restante do pulmão esquerdo; a APD tinha arborização normal; em 2 pacientes, a APD irrigava somente o lobo médio e alguns dos segmentos do lobo inferior; o restante do pulmão direito era irrigado por colaterais sistêmico-pulmonares; a APE tinha arboriza- 




Fig. 2 - Representaçāo esquemática da técnica operatória, no primeiro paciente do Grupo C. A. ressecçāo do segmento estenótico e anastomose entre a artéria pulmonar esquerda e o tronco pulmonar. B: interposiçāo da artéria subclávia direita entre o tronco pulmonar e a artéria pulmonar esquerda intra-hilar.

ção aparentemente normal; em 2 pacientes, a APD se ramificava em segmentos do lobo inferior direito, e o lobo médio e superior direitos eram dependentes de colaterais aortopulmonares. A APE tinha arborização quase normal, existindo somente uma pequena colateral para um segmento inferior.

Em 1 paciente, não existiam evidências angiográficas de APD extraparenquimatosa; as arté rias lobares eram conectadas a três colaterais sistêmico-pulmonares retrobrônquicas, duas das quais com estenose na origem. A APE, nascendo do ducto, tinha arborização aparentemente normal.

\section{Técnica operatória}

Todas as operações foram realizadas sem auxílio de circulação extracorpórea. O lado da toracotomia foi o correspondente ao pulmão com circulação de origem multifocal, ou seja, onde existiam colaterais e a artéria pulmonar.

Em todos os casos, as colaterais foram dissecadas extensamente, até sua entrada no hilo pulmonar, em geral em posição retrobrônquica. A existência de comunicações entre as artérias lobares com origem na artéria pulmonar e as artérias lobares com origem nas colaterais foi investigada, ob- servando-se o fluxo sangüíneo após o pinçamento sucessivo de suas respectivas fontes de origem. Em 6 pacientes, foi realizada anastomose de uma grande colateral com a artéria pulmonar, em posição justa-hilar. Em 2 pacientes, duas grandes colaterais foram anastomosadas entre sí, após comprovar que uma delas tinha comunicação intrapulmonar com a artéria pulmonar. Em 1 paciente, uma colateral foi anastomosada, término-terminalmente em outra colateral de diâmetro maior, que, por sua vez, foi anastomosada término-lateralmente na artéria pulmonar.

Em todos os casos, após a "unifocalização" da circulação pulmonar, as colaterais foram ligadas proximalmente e a operação de Blalock-Taussig, modificada com tubo de $5 \mathrm{~mm}$ (5 casos) e de $6 \mathrm{~mm}$ (4 casos), foi realizada.

Tipo C: Todos os segmentos broncopulmonares conectados com colaterais aortopulmonares (sem tronco pulmonar, ou AP's extraparenquimatosas).

Dois pacientes, 1 com dezoito meses de idade e outro com oito anos, foram submetidos a correção cirurgica; o primeiro em três etapas e o segundo em duas. 
O primeiro paciente, com insuficiência cardíaca e cianose, apresentava, angiograficamente, anatomia típica de atresia pulmonar com CIV; não foi possivel demonstrar tronco pulmonar ou AP's extraparenquimatosas.

A circulação pulmonar era exclusivamente dependente de quatro grandes colaterais (Figura 3), que nasciam da aorta torácica descendente.

O tratamento cirúrgico foi planejado em três estágios:

10) Através de toracotomia esquerda, a aorta descendente foi dissecada; após isto, a pleura visceral foi aberta no hilo pulmonar e as veias pulmonares foram afastadas anteriormente. Foi, então, possivel identificar artérias lobares intraparenquimatosas. Para construir um novo segmento arterial extraparenquimatoso, que pudesse permitir, posteriormente, a reconstrução da continuidade entre o ventrículo direito e as artérias lobares, um tubo de PFTE de $10 \mathrm{~mm}$ de diâmetro foi preparado, deixando-o com a forma de um funil de $25 \mathrm{~mm}$ de comprimento. Este tubo foi anastomosado, na sua base, a eixo longitudinal da maior artéria lobar. $\mathrm{Na}$ outra extremidade do tubo, com menor diâmetro, um outro tubo, de $5 \mathrm{~mm}$ de diâmetro, foi anastomosado e a outra extremidade foi suturada, término-lateralmente à artéria subclávia esquerda. As colaterais foram, então, ligadas. O estudo angiográfico, através de injeção no Blalock, demonstrou bom fluxo para as artérias intrapulmonares esquerdas.
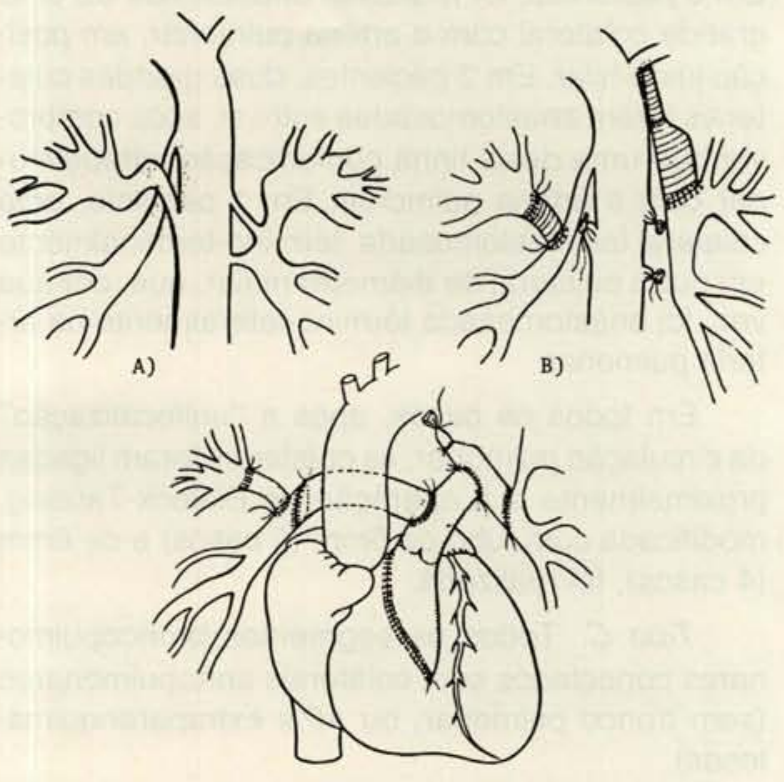

C)

Fig. 3 - A: Representação esquemática do suprimento arterial pulmonar. B: situação após o primeiro e o segundo estágio. C: restabelecimento da continuidade ventricular direita-circulaçāo pulmonar, após o fechamento da comunicaçāo interventricular.
2.) (Um mês depois): Através de toracotomia direita, as colaterais foram dissecadas. Para anastomosá-las entre sí, foi necessário interpor segmento de veia ázigos (após ressecar as válvulas da veia). Isto porque o brônquio direito estava interposto entre os dois vasos. A colateral inferior foi deixada com sua conexão aórtica intacta.

3.) (Quatro meses depois): Através de esternotomia mediana, o coração foi exposto, não se encontrando evidências de tronco pulmonar, ou AP's.

O hilo pulmonar esquerdo foi abordado e o tubo em forma de bota, previamente anastomosado às artérias lobares, exposto. Após isto, a colateral inferior foi dissecada, à direita da aorta e atrás da veia cava superior. Com circulação extracorpórea a 20: C e hipofluxo, a face anterior do tubo em forma de bota foi ressecada, resultando em uma janela de $12 \mathrm{~mm}$ de diâmetro. Um tubo valvulado e pré-coagulado de $14 \mathrm{~mm}$ foi, então, anastomosado nesta janela. Após isto, outro tubo de $8 \mathrm{~mm}$ de diâmetro foi anastomosado, término-lateralmente, nesta colateral; a outra extremidade deste tubo foi anastomosada, término-lateralmente, no tubo valvulado. Uma incisão longitudinal no ventrículo direito foi, então, realizada. Não havia infundíbulo. $\mathrm{A}$ anatomia da CIV e do VD foi similar à encontrada em casos de truncus arteriosus. A CIV foi fechada com retalho de pericárdio bovino, deixando a aorta dextroposta, conectada ao ventrículo esquerdo. A extremidade biselada do tubo foi, então, anastomosada no ventrículo direito. A anastomose de Blalock modificado foi ligada.

O segundo paciente apresentava três colaterais para o pulmão direito e uma grande colateral para o esquerdo, todas nascendo da aorta torácica descendente. Não tinha evidências de tronco ou artérias pulmonares extraparenquimatosas. A correção cirúrgica foi planejada em duas etapas. $\mathrm{Na}$ primeira, realizada através de toracotomia direita, foi feita anastomose término-terminal entre as duas maiores colaterais e um tubo em forma de bota, de maneira análoga à descrita anteriormente, foi anastomosado às artérias lobares intraparenquimatosas, em posição ântero-superior em relação ao brônquio direito, no pós-operatório imediato. $\mathrm{O}$ estudo angiográfico, realizado através do Blalock modificado, mostrou bom fluxo para as artérias lobares. A segunda etapa foi realizada quatro meses após. Um tubo de $10 \mathrm{~mm}$ foi anastomosado à face anterior do tubo em forma de bota, colocado previamente no hilo do pulmão direito; outro tubo de $18 \mathrm{~mm}$, valvulado, foi anastomosado, distalmente à grande colateral esquerda, na altura do hilo. $\mathrm{O}$ primeiro foi anastomosado ao segundo, término-la- 




Fig. 4 - Representaçāo esquemática da técnica operatória realizada no segundo paciente do Grupo C. A: suprimento arterial pulmonar: nota-se a disposiçāo das colaterais direitas em relação ao brônquio direito. B: a situação após o primeiro estágio. C.: restabelecimento da continuidade ventriculo direito-circulação pulmonar:

teralmente, em posição supravalvar. A extremidade proximal do segundo tubo foi anastomosada, após fechamento da CIV, no ventrículo direito (Figura 4).

\section{RESULTADOS}

19) Tipo A: Todos os segmentos broncopulmonares conectados às AP's centrais (Tabela 1).

$A_{1}$ ) Com artérias pulmonares "normais" (os resultados foram considerados previamente).

$\mathrm{A}_{2}$ ) Com artérias pulmonares näo contluentes, ou estenóticas. Houve um óbito imediato $(11,1 \%)$ e nenhum no pós-operatório tardio. O paciente faleceu subitamente, no $5^{\circ}$ dia de pós-operatório, sem ter apresentado quadro clínico de hipóxia, ou de insuficiência cardíaca. Todos os sobreviventes foram submetidos a um, ou mais estudos angiográficos pós-operatórios. Dois pacientes deste grupo foram submetidos a correção total da anomalia dois e meio e 4 anos, respectivamente, após a primeira operação. Nos 2 pacientes, a evolução foi satisfatória e o estudo angiográfico pós-operatório demonstrou bom enchimento das artérias pulmonares e ausência de gradientes significantes entre o ventrículo direito e as artérias pulmonares distais.

2.) Tipo B: Alguns dos segmentos broncopulmonares conectados às artérias pulmonares centrais e outras às colaterais aortopulmonares.

TABELA 1

ATRESIA PULMONAR COM CIV 1975 - 1986

Correção Correção em
Tipos Convencional Etapas Mortalidade

1․ 2: 3:

TIPO A $(\mathrm{N}=34)$

a) AP'S "NORMAIS"

TIPO B $(\mathrm{N}=9) \quad-\quad 9 \quad-3 / 1 \quad 1(11,1 \%)$

$\begin{array}{lllllll}\operatorname{TIPO} \mathrm{C}(\mathrm{N}=2) & - & 2 & 1 & 2 & 0(0 \%)\end{array}$

ÍBITOS 
Não houve nenhum óbito, na primeira etapa da operação, de unifocalização da circulação pulmonar associada à operação de Blalock-Taussig. Nos 3 pacientes que foram submetidos em uma segunda etapa cirúrgica, a correção total da anomalia, 1 paciente faleceu no 30 : dia de pós-operatório, de septicemia após mediastinite. Os pacientes restantes tiveram boa evolução. O estudo angiográfico pós-operatório imediato, realizado em todos os pacientes, demonstrou bom funcionamento da anastomose de Blalock e adequado enchimento das artérias lobares.

3:) Tipo C: Todos os segmentos broncopulmonares conectados às colaterais sistêmico-pulmonares.

Os dois pacientes tiveram boa evolução clínica. O estudo angiográfico pós-operatório do primeiro paciente demonstrou ausência de comunicação interventricular e fluxo adequado para as artérias lobares esquerdas e para o lobo médio, inferior e segmentos do lobo superior do pulmão direito. Os segmentos do lobo superior direito, correspondentes à colateral superior direita, não foram contrastados. Apesar disto, as pressões permaneceram baixas: no tubo, acima da valva $34 / 4 \mathrm{mmHg}$, sem insuficiência valvar; ventrículo direito $62 / 4$, sendo a pressão arterial sistêmica $94 / 63 \mathrm{mmHg}$.

O estudo angiográfico pós-operatório do segundo paciente demonstrou ausência de comunicaçōes intracardíacas e adequado enchimento das artérias lobares dos dois pulmões. Houve moderada insuficiência pulmonar. As pressões foram: no tubo, acima da valva, $38 / 12 \mathrm{mmHg}$; no ventrículo direito, $68 / 12$, sendo a pressão arterial sistêmica de $104 / 75 \mathrm{mmHg}$. A evolução clínica foi boa, apenas com uso de cardiotônicos.

\section{DISCUSSĀO}

\section{Reconstrução de artérias pulmonares estenó- ticas, ou não confluentes}

Os pacientes com atresia pulmonar e CIV apresentam, com freqüência, estenoses, ou descontinuidade das artérias pulmonares $4,5,6,10,18$. Estas malformações ocorrem devido às diferentes origens embriológicas do tronco pulmonar, das ártérias pulmonares e de seus ramos intraparenquimatosos.

O tratamento simultâneo do estado hipóxico através de anatomose sistêmico-pulmonar e da correção das anomalias das AP's é baseado nos seguintes conceitos: a) o sangue desviado para o pulmão é mais uniformemente distribuído para ambos os pulmões, evitando a predominância do fluxo exclusivamente para o pulmão ipsilateral e lesões vasculares tardias ${ }^{8 .}{ }^{16}$; b) mantendo adequado fluxo para o pulmão contralateral, o crescimento das estruturas vasculares e bronco-alveolares poderá ser mais próximo do normal ${ }^{3}$; c) a possibilidade de correção cirúrgica futura é aumentada.

As técnicas empregadas variaram conforme o tipo de malformação e a experiência cirúrgica. A anastomose sistêmico-pulmonar, realizada como primeiro passo cirúrgico, permitiu manter adequada oxigenação sistêmica e o isolamento da área a ser corrigida, sem necessidade de circulação extracorpórea.

Baseados nos resultados obtidos, acreditamos que, em crianças com indicação de anastomose de Blalock-Taussig, as anomalias das artérias pulmonares, quando presentes, devem ser corrigidas simultaneamente.

\section{Abordagem cirúrgica de pacientes com anor- malidades de arborização pulmonar, ou ausên- cia de tronco pulmonar e AP's centrais}

O conceito de que as anomalias de arborização pulmonar são susceptiveis de correção cirúrgica é baseado nas seguintes premissas: a) as artérias lobares, segmentais e acinais são, na maioria dos casos, essencialmente normais, em estrutura e em função, sendo adequadas para as trocas gasosas. Estas artérias podem ser dissecadas dentro do pulmão e unificadas, com a construção de um segmento arterial intermediário. Isto adquire singular importância, quando as colaterais sistêmico-pulmonares não podem ser usadas cirurgicamente, por serem finas, tortuosas e/ou estenóticas; b) quando as colaterais são grandes, a circulação arterial pulmonar pode ser unificada, anastomosando-as entre sí, ao nível do hilo.

Para os casos de ausência de tronco e artérias pulmonares extraparenquimatosas, a técnica proposta em estágios deu resultados satisfatórios. $\mathrm{O}$ estudo angiográfico, após cada procedimento, permite melhor avaliação da anatomia arterial pulmonar, assim como uma correta avaliação do risco cirúrgico da correção final (estabelecimento da continuidade entre o ventrículo direito e a circulação pulmonar).

No primeiro paciente deste grupo (tipo C), a falta de enchimento de alguns dos segmentos do lobo superior direito poderia ser explicada por dois fatores: a) a colateral correspondente, não estenótica, poderia ter transmitido, integralmente, a pressão sistêmica aórtica, resultando, provavelmente, em aumento de resistência vascular e subseqüente 
BARBERO-MARCIAL, M.; ATIK, E.; RATTI, M.; KAJITA, L. J.; RISO, A.; VERGINELLI, G.; BITTENCOURT, D.; PILEGGI, F. : JATENE, A. D. - Novas técnicas cirúrgicas para o tratamento da atresia pulmonar com comunicação interventricular e anomalias de artérias pulmonares incluindo o assim chamado truncus tipo IV. Rev. Bras. Cir. Cardiovasc., 2(1):22-31, 1987.

trombose da anastomose. Em casos similares, achamos conveniente, agora, estudos pressóricos das colaterais sistêmico-pulmonares, para poder avaliar a presença da doença obstrutiva vascular segmentar; b) a escolha da veia ázigos, como um substituto arterial pulmonar, poderia ter sido inadequada. Apesar disto, a relação do pico sistólico ventricular direito/ventricular esquerdo foi inadequada. Isto é uma evidência de que o leito vascular pulmo- nar, conectado ao ventrículo direito, é suficientemente grande para aceitar todo o volume minuto cardiaco. Estes pacientes do grupo $\mathrm{C}$ encontramse, agora, no grupo dos pacientes portadores de tubos extracardiacos, com as bem conhecidas complicaçōes que isto implica. No entanto, os pacientes mudaram de uma condição não operável para um grupo com melhor qualidade e expectativa de vida.

RBCCV

BARBERO-MARCIAL, M.; ATIK, E.; RATTI, M.; KAJITA, L.J.; RISO, A.; VERGINELLI, G.; BITTENCOURT, D.; PILEGGI. F.; JATENE, A.D. - New surgical techniques for treatment of pulmonary atresia with ventricular septal defect and pulmonary arteries anomalies including the so-called tipo IV truncus. Rev. Bras. Cir. Cardiovasc., 2(1):22-31, 1987

ABSTRACT: Fourty-two patients with pulmonary atresia and interventricular septal defect were submited to a partial or total correction, between January 1975 and October 1986, with a range of 2 months to 18 years of age. Three groups were identified: Group A: 34 patients with all bronco-pulmonary segments connected to pulmonary arteries (PA); Group B: 6 patients with some of the bronco-pulmonary segments connected to PA's; Group C: 2 patients with all bronco-pulmonary segments connected to systemic pulmonary collaterals. One to three stages were utilized to perform the proper correction. In Group A 17 were treated in one stage, with 3 deaths; 9 were submitted to a first stage where the pulmonary arteries were reconstructed with an additional Blalock-Taussig (BT) shunt, with 1 death. In two of them a second stage for total correction was performed, with no death. In the remaining 8 patients the collateral circulation was ligated and a BT shunt performed, with 1 hospital death. In Group B the first stage joining the intra or extra-pulmonary collaterals was obtained in 6 cases, without death. In two of them a total correction was performed, with 1 death. In group $\mathrm{C}$ a three stage operation was done in 1 patient. In the first stage an intermediate arterial segment was constructed between the lobar arteries and the BT shunt. In the second stage the contra-lateral collaterals were joined. The third stage was the establishment of the continuity between the right ventricle and the reconstructed pulmonary circulation with a good result. In the other patient of this group, until now, only the first stage was done. Serial hemodynamic studies were carried out in 32 patients. These proposed techniques demonstrate the possibility of total correction with appropriate righ-to-left ventricular pressure relation.

DESCRIPTORS: pulmonary atresia, surgery; ventricular septal defect, surgery; pulmonary arteries, anomalies, surgery.

\section{REFERÊNCIAS BIBLIOGRÁFICAS}

1 ALFIERI, O.; BLACKSTONE, E.H.; KIRKLIN. J.W.; PACIFICO, A.D.; BARGERON Jr., L.M. - Surgical treatment of tetralogy of Fallot with pulmonary atresia. J. Thorac. Cardiovasc. Surg., 76 (3): 321-335, 1978.

2 BARBERO-MARCIAL, M.; RISO, A.; LOPES, A. A. B.; BITTENCOURT, D.; AULER, J. O. A.; JATENE, A. D. Correction of pulmonary atresia with ventricular septal defect in the absence of the pulmonary trunk and the central pulmonary anteries (so called truncus type IV). J. Thorac. Cardiovasc. Surg., em publicação,

3 DE TROYER, A.; YERNAULT, J. C.; ENGLERT, M. Lung hypoplasia in congenital pulmonary valve stenosis. Circulation, 56 (4): 647-651, 1977.

4 FRANCH, R. H. \& GAY, B. B. - Congenital stenosis of the pulmonary artery branches: a classification, with postmortem findings in two cases. Am. J. Med., 35 (1): 512-529, 1963.

5 GASUL, B. M.; ARCILLO, R. A.; LEV, M. B. S. - Heart disease in children: diagnosis and treatment. New York, J. B. Lippicott Company, 1966.
6 GAY, B. B.; FRANCH, R. H.; SHUFORD, W. H.; ROGERS, J. V. - Roentgenologic features of simple and multiple coarctations of the pulmonary artery and branches. $\mathrm{Am}$. J. Roentgenol., 90 (3): 599-612, 1962.

7 GILL, O. C.; MOODIE, D. S.; McGOON, D. C. - Staged surgical management of pulmonary atresia with diminutive pulmonary arteries. J. Thorac. Cardiovasc. Surg., 73 (3): 436-442, 1977.

8 HOFSCHIRE, P. J.; ROSENQUIST, G. C.; RUCKERMAN, R. N.; MOLLER, J. H.; EDWARDS, J. E. - Pulmonary vascular disease complicating the Blalock-Taussig anastomosis. Circulation, 56 (1): 124-126, 1977.

9 JEFFERSON, K.; REES, S.; SOMERVILLE, J. - Systemic arterial supply to the lungs in pulmonary atresia and its relation to pulmonary artery development. Br. Heart J., 34 (4): 418-427, 1972.

10 McCUE, C. M.; ROBERTSON, L. W.; LESTER. R. G.; MAUCK Jr., H. P. - Pulmonary artery coarctations: a report of 20 cases with review of 319 cases from the literature.J. Pediatr., 67 (1): 222-238, 1965. 
11 McGOON, D. C.; BAIRD, D. K.; DAVIS, G. D. - Surgical management of large bronchial collateral arteries with pulmonary stenosis of atresia. Circulation, 52 (1): 109-118, 1976.

12 MURPHY, D. A.; SRIDHARA, K. S.; NANTON, M. A.; ROY, D. L.; BELCOURT, C. L.; GILLIS, D. A. - Surgical correction of pulmonary atresia with multiple large systemic pulmonary collaterals. Ann. Thorac. Surg., 27 (5): 460-464, 1979.

13 NIHILL, M. R.; MULLINS, C. E.; McNAMARA, D. G. Visualization of the pulmonary arteries in pseudotruncus by pulmonary vein wedge angiography. Circulation, 58 (1): 140-147, 1978.

14 PACIFICO, A. D.; ALLEN, R. H.; COLVIN, E. V. - Direct reconstruction of pulmonary artery arborization anomaly and intracardiac repair of pulmonary atresia with ventricular septal defect. Am. J. Cardiol., 55 (13): 1647-1649, 1985.

15 RANKIN, J. S.; STERBA, R. J.; KARIS, J. H.; OHLDHAM Jr., H. N. - Staged correction of pulmonary atresia and absent right pulmonary artery. Arch. Surg., 118 (8): 974-978, 1983.

16 SHAPIRA, N.; ROSENTHAL, A.; HEIDELBERGER, K.; BADANOWSKI, R.; BEHRENDT, D. - Pulmonary vascular morphology in shunted and nonshunted patients with tetralogy of Fallot. J. Thorac. Cardiovasc. Surg., 83 (5): 650-658, 1982.

17 SOTO, B.; PACIFICO, A. D.; LUNA, R. F.; BARGERON Jr., L. M. - A radiographic study of congenital pulmonary atresia with ventricular septal defect. Am. J. Roentgenol., 129 (6): 1027-1037, 1977.

18 WEINBERG, W.; AGUSTSSON, M. H.; D'CRUZ, I. A.; BICOFF, J. P.; BEBRAVESH, M.; RAFFENSPERGER, J. G.; FELL, E. H. - Stenosis of the branches of the pulmonary artery. J. Thorac. Cardiovasc. Surg., 47 (1): 40-49, 1964.

\section{Discussão}

\section{DR. PAULO RODRIGUES DA SILVA}

Rio de Janeiro, RJ

Inicialmente, quero cumprimentar o Dr. Miguel Barbero-Marcial, pela qualidade dos resultados cirúrgicos obtidos, com cardiopatia congênita de diffcil correção e alto risco cirúrgico, até chegar-se à sua correção completa, ou total, não somente pelos problemas próprios da árvore arterial pulmonar intra e extraparenquimatosa, que acompanham, em alto percentual, esta patologia, como também, pela necessidade, na maioria dos casos, de se fazerem cirurgias em várias etapas, para que, em casos selecionados, se chegue à correção total, ou seja, conecção VD / A P fisiologicamente adequada em termos de relação $p V D / p V E$ (igual ou menor que 0,85). Assim sendo, quero ressaltar o expressivo baixo índice de mortalidade média geral obtido pelos autores, neste grupo de pacientes, que foi de $13,7 \%$. Quero ressaltar, também, o alto índice de pacientes deste mesmo grupo e que chegaram à correção total e que foi de $58,8 \%$. Uma dúvida, apenas, nos ficou, sobre o apresentado pelo autor e colaboradores, e se refere à conclusão deste trabalho. Concluem os autores "que as técnicas cirúrgicas utilizadas permitem obter condições para correção completa desta patologia, com adequada relação pós-operatória das pressōes pV D / pV E". De um modo geral, certamente nos pacientes do tipo $A$ e em muitos (maioria) do tipo $\mathrm{B}$, concordamos com a conclusão dos autores. No entanto, uma ressalva, no nosso entender, deve ser adicionada a esta afirmação, especificamente relacionada ao tipo $\mathrm{C}$, qual seja: independentemente da qualidade das técnicas empregadas e conhecidas na 1a etapa da correção, seja por técnicas de shunt, ou por "unifocalização" das fontes de suprimento sangüíneo pulmonar, especificamente no tipo $\mathrm{C}$ do autor, apesar da já reconhecida melhora sintomatológica e de sobrevida pós-operatória, observada nestes pacientes com as cirurgias paliativas, este resultado ideal, acima mencionado e obtido de forma brilhante pelos autores em um de seus dois casos do tipo C, quando da correção total, deverá, na nossa opinião, ser obtido num percentual em torno de $70 \%$ dos casos deste tipo de pacientes que vierem a ser operados (o que, reconheço, não deixa de ser um grande e importante avanço), pelos seguintes motivos: (Slide) Alfieri, Kirklin, Pacífico e colaboradores, em mais de uma publicação, demonstraram que os pacientes que tinham o suprimento sangüíneo pulmonar funcional proveniente de colaterais apenas do tipo sistêmico-pulmonar, na sua grande maioria tiveram, no pós-operatório, o índice de relação $\mathrm{pV} D$ / $\mathrm{pV}$ E muito maior (acima de 0,80 ) que o obtido entre os pacientes que tinham outras fontes de fluxo sangüineo pulmonar. Em adição, neste grupo de pacientes, houve uma percentual grande de disfunção pulmonar ( $31 \%$, em média), além de severos problemas intraparenquimatosos dos chamados defeitos de arborização ( $38 \%$, em média). (Slide) Além dos problemas acima mencionados, que podem ocorrer, tanto no tipo B como no C, porém, estatística e severamente mais importantes entre os pacientes do tipo $\mathrm{C}$, também são descritos, neste último grupo, problemas de "compartimentalização" da circulaçãc lobar e segmentar e de estenoses vasculares parenquimatosas em vários níveis de distanciamento do hilo pulmonar, aparentemente de difícil acesso cirúrgico. Admito, no entanto, que estas observações possam, para alguns, ser consideradas con- 
BARBERO-MARCIAL, M.; ATIK, E.; RATTI, M.; KAJITA, L. J.; RISO, A.; VERGINELLI, G.; BITTENCOURT, D.; PILEGGI, F. : JATENE, A. D. - Novas técnicas cirúrgicas para o tratamento da atresia pulmonar com comunicação interventricular e anomalias de artérias pulmonares incluindo o assim chamado truncus tipo IV. Rev. Bras. Cir. Cardiovasc., 2(1):22-31, 1987.

jecturais, porém acredito ser, com base no que se sabe, até hoje, da literatura. Espero, no entanto, que não se confirmem, com o aumento da casuística dos autores, com os pacientes do tipo C; dai, mais um motivo para elogiar o esforço, a elegância, a inteligência e a confiança demonstrada pelos autores, para, com o seu trabalho, provarem o contrário. Muito obrigado.

DR. FERNANDO ANTÓNIO FANTINI Belo Horizonte, $M G$

Primeiramente, gostariamos de parabenizar o Dr. Miguel e seu grupo, não só pelo excelente material que nos apresentou, como pela efetiva contribuição ao tratamento dessa patologia, de abordagem cirúrgica muitas vezes complexa, em vista da grande variação anatômica da arborização pulmonar. A experiência do nosso grupo, na Santa Casa e Hospital Biocor de Belo Horizonte, refere-se a 23 pacientes nos quais realizamos 24 cirurgias paliativas e 4 correçōes totais. $A$ idade variou de 2 dias a 18 anos, sendo que 16 pacientes tinham menos de 6 meses e 10 ainda se encontravam no periodo neonatal. Em vista dessa faixa etária baixa e do quadro clínico em geral grave desses pacientes, todos foram abordados inicialmente de forma simplificada, através de realização de anastomoses sistêmico-pulmonares, que possibilitaram uma estabilização adequada do quadro clínico, permitindo, ainda, estudos hemodinâmicos e angiográficos mais detalhados da circulação pulmonar. Nos pacientes do tipo A sem alterações nas artérias pulmonares da classificação do Dr. Miguel, cremos que a conduta está bem estabelecida, não havendo necessidade de maiores comentários. Nos casos com estenoses importantes de artéria pulmonar, não realizamos plastias destas artérias em nenhum dos nossos pacientes, sendo o Blalock realizado no lado oposto ao do canal, evitando-se, assim, a interrupção do suprimento sangüíneo ao pulmão melhor vascularizado durante a cirurgia. A observação de 2 desses casos operados dentro do 1 ? mês de vida mostrou que a presença dessa estenose não impediu o crescimento normal das artérias pulmonares, sendo que, nesse paciente, a estenose foi abordada durante a correção total, sem maiores problemas. Nos pacientes com artérias pulmonares não confluentes, implantamos o Blalock no lado oposto ao do canal arterial. No entanto, os pacientes dos grupos $\mathrm{B} \mathrm{e} \mathrm{C}$ da presente classificação constituem, sem dúvida, o grande desafio dessa patologia. Assim, a presente técnica desenvolvida pelo $\mathrm{Dr}$. Miguel e seu grupo, centralizando a circulação pulmonar e criando condições para a correção total, deve ser encarada com otimismo. por oferecer possibilidade de tratamento para pacientes de difícil manuseio. Outro ponto que gostariamos de abordar é a respeito dos condutos valvulados comumente empregados na correção definitiva desses pacientes. Em vista das complicaçōes com os tubos de Dacron, relatados freqüentemente na literatura, temos utilizado, nos nossos casos. um conduto valvulado biológico, em que uma válvula sem anel de sustentação é suturada no interior de um segmento de veia ilíaca bovina. Já usamos este tipo de tubo em 5 dos nossos pacientes, 3 deles portadores de atresia pulmonar com CIV, com bom resultado em um seguimento de até 3 anos. Temos 2 perguntas a fazer ao Dr. Miguel: Qual é a sua expectativa em relação à evolução tardia desses pacientes do Grupo C em vista de: 1) uso de múltiplas próteses; 2) possibilidade de hipertensão pulmonar residual, descrita em outras técnicas de unifocalização da circulação pulmonar e não observadas na sua experiência.

\section{DR. BARBERO-MARCIAL}

(Encerrando)

Agradeço os comentários feitos ao nosso trabalho. Com relação à percentagem de pacientes do tipo $\mathrm{C}$ que poderão ser beneficiados com esse tipo de operação, acreditamos que seja muito alta. Embora estejamos iniciando a aplicação das técnicas descritas, o nosso parecer é que, com a abordagem em etapas, as anormalidades de arborização podem ser corrigidas sucessivamente, primeiro em um hemitórax, depois no outro e, finalmente, após nova e definitiva análise angiográfica, a reconstrução de continuidade entre o ventrículo direito e as "novas pulmonares" poderá ser realizada. Respondendo às perguntas do Dr. Fantini, é um fato bem conhecido que os pacientes portadores de tubos valvulados apresentam, em certa percentagem, problemas tardios. No entanto, gostaria de chamar atenção para o fato de que estes pacientes com a técnica utilizada foram retirados de um grupo considerado inoperável e de mau prognóstico, e integrados a outro grupo de pacientes que, embora sejam portadores de próteses valvulares, têm uma indubitável melhor expectativa de vida. Quanto à hipertensão pulmonar residual, em nenhum dos nossos pacientes este fenômeno foi observado. 